\title{
A Projector-Camera System with Real-Time Photometric Adaptation for Dynamic Environments
}

\author{
Kensaku Fujii \\ NTT Cyber Space Laboratories \\ NTT Corporation \\ Yokosuka, JAPAN \\ fujii.kensaku@lab.ntt.co.jp
}

\author{
Michael D. Grossberg \\ Dept. Computer Science \\ City University of New York \\ New York, USA \\ grossberg@cs.ccny.cuny.edu
}

\author{
Shree K. Nayar \\ Dept. Computer Science \\ Columbia University \\ New York, USA \\ nayar@cs.columbia.edu
}

\begin{abstract}
Projection systems can be used to implement augmented reality, as well as to create both displays and interfaces on ordinary surfaces. Ordinary surfaces have varying reflectance, color, and geometry. These variations can be accounted for by integrating a camera into the projection system and applying methods from computer vision. The methods currently applied are fundamentally limited since they assume the camera, projector, and scene are static. In this paper, we describe a technique for photometrically adaptive projection that makes it possible to handle a dynamic environment.

We begin by presenting a co-axial projector-camera system whose geometric correspondence is independent of changes in the environment. To handle photometric changes, our method uses the errors between the desired and measured appearance of the projected image. A key novel aspect of our algorithm is that we combine a physicsbased model with dynamic feedback to achieve real time adaptation to the changing environment. We verify our algorithm through a wide variety of experiments. We show that it is accurate and runs in real-time. Our algorithm can be applied broadly to assist HCI, visualization, shape recovery, and entertainment applications.
\end{abstract}

\section{Camera Assisted Projection}

The recent availability of cheap, small, and bright projectors has made it practical to use them for a wide range of applications such as creating large seamless displays $[8,11,12$, $25]$ and immersive environments [5, 21]. By introducing a camera into the system, and applying techniques from computer vision, the projection system can operate taking its environment into account. For example, it is possible to allow users to interact with the projected image creating projected interfaces $[1,10,24]$. The camera can assist projection by taking into account distortions due to surface geometry [18,
19], or eliminate shadows cast on the projected image [9, 22]. The camera can also assist projection by color correcting a homogeneous colored surface [6], or by correcting for spatially varying color and texture [14,3]. Related methods have been applied to improve the recovery of 3D geometry from structured light [4], to neutralize the appearance of a painting [2], to restore color-damaged paintings [26], and to control the appearance of 3D objects [7].

All previous work that takes into account both geometric and photometric properties of projection has assumed both a static scene and projection system. The assumption that the scene and system remain static is very restrictive. This is especially true when we consider recently presented applications that require hand-held or mobile projection systems such as iLamps and RFIG Lamps [18, 20]. Even when the projector is not mobile, there are systems that use computer-controlled motors to steer the projected image onto any surface in an environment [13, 16]. For applications in which the projector is fixed, the scene may be dynamic, such as with illuminating clay (a 3D tangible interface) [17]. It is thus critical for projectorcamera systems to be able to take into account both geometric and photometric changes.

One approach that was proposed to handle photometric changes is direct dynamic feedback from the camera for each pixel [14]. This method requires several frames to converge, limiting its use to projecting static images on static scenes. A method for projection of video on static scenes was proposed in $[7,14]$ using a photometric model. The parameters of this model are determined off-line by projecting and capturing a sequence of calibration images. Arbitrary images (video) are then compensated on-line, before projection, using the same model; the model is valid as long as the scene remains static. Whenever the scene changes, however, the calibration images must be re-projected making this method impractical in a dynamic environment.

In this work, we present a novel hybrid method which combines a model based approach with dynamic feedback 
to adapt photometric compensation for projection in dynamic environment. The model we introduce is an approximation that separates the effects of the projector and camera from those of the scene. We present a method that, after a one-time initial calibration, can adapt the model using only the errors between the desired and measured appearance of the projected image. Unlike vision methods such as optical flow, we do not rely on spatial variations in the reflectance of the surface or the presence of features. This purely photometrically based method allows us to determine the correct adaptation in a single frame, providing a rapid means to detect and adapt to apparent changes in surface color. This allows projection of video on a dynamic scene. We also provide a simple means to account for the surface shape when determining the correct image to project. Previous projection methods either assumed the surface shape to be known or had to recover it actively. This is particularly difficult for a dynamic scene. We present a projection system whose geometry is independent of the surface. Thus, after making a single off-line geometric calibration, the geometry remains correct even as the environment changes.

\section{Projector-Camera Correspondence}

A fundamental assumption we make is that the camera is a proxy for the viewer. That is, if the image looks correct to the camera, we will assume it looks correct to a viewer. Hence, our goal is to be able to determine the input brightness at a given pixel to achieve a desired captured brightness. As a first step we must determine the geometric mapping between points in the projected image and the captured image. Determining this relationship, in general, is difficult. The $3 \mathrm{D}$ shape of the surface can create parallax between the projector and camera, which will affect the map. More seriously, due to occlusion or non-overlapping fields of view, parallax between projector and camera can make correspondence impossible.

We avoid these correspondence problems altogether, by designing a system in which the mapping between the projected and captured images is scene-independent. We accomplish this by making the optics of the projector and the camera coaxial. Note that a similar approach was taken for the I/O bulb (co-located projector and camera) [24]. Our compact system is designed to be a simple attachment to an off-the-shelf projector. We place the beam-splitter in front of both the camera and projector lenses, as shown in Fig. 1(a). A prototype system using a FireWire camera is shown in Fig. 1(b). This configuration ensures that all surfaces visible to the camera can also be projected upon; there is no possibility of occlusion and no parallax. To compute the correspondence between the camera and the projector, a sequence of binary coded images is projected and captured. Since the geometry of the system does not depend on the

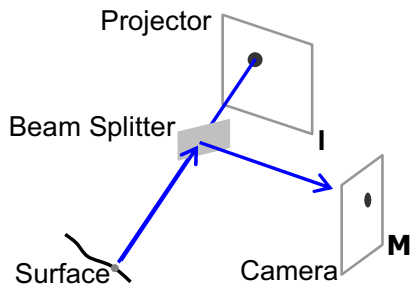

(a)

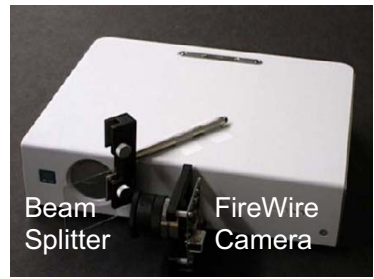

(b)
Figure 1: A coaxial projector-camera system. (a) A schematic of the system. (b) A prototype system.

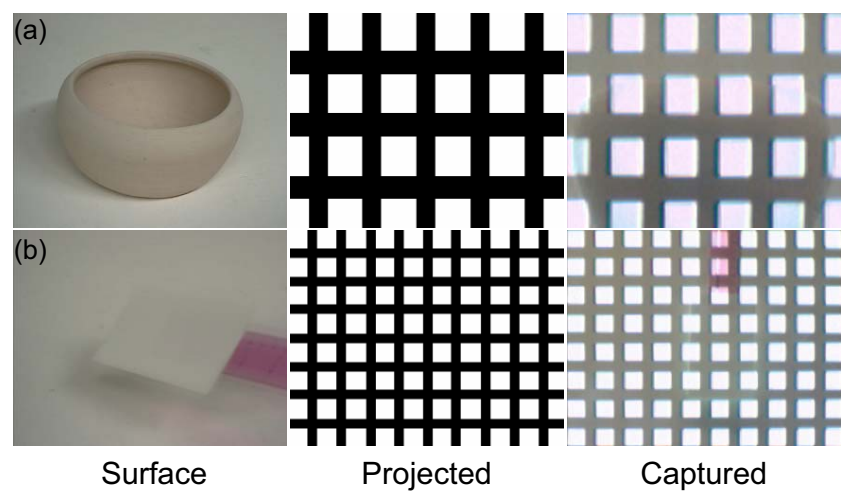

Figure 2: Verification of geometric scene-independence. (a) A test pattern projected onto a curved vase. (b) One frame from a sequence in which an object moves.

scene, once the system is calibrated, it does not need to be re-calibrated.

We verified that our system is scene-independent by projecting and capturing binary coded checkerboard patterns. The coding allowed us to rapidly obtain the correspondences for all points. Fig. 2(a) shows results for a pattern projected onto a curved vase. The captured image is essentially identical to the projected pattern, even though points of the surface have varying depths from the system. The resulting RMS error of the recovered mapping was less than 0.54 pixels. Thus we obtain an accurate projectorcamera correspondence independent of the scene structure. In Fig. 2(b) we see one frame of a sequence where an object in the scene is moved around. Again, the alignment is not effected by the motion. If the projector-camera system is moved then our system's geometric correspondence is also preserved. Thus, by a simple modification of the optics we have shown that we can make the system's geometry independent of the dynamic environment.

\section{Photometric Model}

We assume that the measured pixel value for a given channel is linear in irradiance and that the projector brightness 
$P_{K}$ is linear in its input. ${ }^{1}$ The brightness $P_{K}$ is modulated by the spectral response $w_{K}(\lambda)$ for projector channel $K$ where $\lambda$ is wavelength. If $f(\lambda)$ is the irradiance on the scene due to the environmental lighting then the irradiance measured by the sensor is

$$
C_{L}=\int\left(f(\lambda)+P_{K} w_{K}(\lambda)\right) s(\lambda) q_{L}(\lambda) d \lambda,
$$

where $s(\lambda)$ is the spectral reflectance of the surface and $q_{L}(\lambda)$ is the camera spectral response for color camera channel $L$. The integration is done over the visible spectrum, as in [7, 14]

We make a key approximation to the above model that will allow us to adapt to dynamic surfaces. Suppose that the projector and the camera each have three color channels (RGB). Our main assumption is that the reflectance of the surface is effectively constant within each of the camera bands so that

$$
C_{L} \simeq A_{L} \int\left(f(\lambda)+P_{K} w_{K}(\lambda)\right) q_{L}(\lambda) d \lambda .
$$

The spectral curves recovered in [23] indicate that this assumption is reasonable for many ordinary surfaces. ${ }^{2}$ Using equation (2), we can write the model at each pixel compactly using vectors and matrices as

$$
\mathbf{C}=\mathbf{A}(\mathbf{F}+\mathbf{V} \mathbf{P})
$$

where:

$$
\begin{gathered}
\mathbf{C}=\left[\begin{array}{c}
C_{R} \\
C_{G} \\
C_{B}
\end{array}\right], \mathbf{A}=\left[\begin{array}{ccc}
A_{R} & 0 & 0 \\
0 & A_{G} & 0 \\
0 & 0 & A_{B}
\end{array}\right], \mathbf{F}=\left[\begin{array}{c}
F_{R} \\
F_{G} \\
F_{B}
\end{array}\right], \\
\mathbf{V}=\left[\begin{array}{lll}
V_{R R} & V_{R G} & V_{R B} \\
V_{G R} & V_{G G} & V_{G B} \\
V_{B R} & V_{B G} & V_{B B}
\end{array}\right], \mathbf{P}=\left[\begin{array}{c}
P_{R} \\
P_{G} \\
P_{B}
\end{array}\right], \\
V_{L K}=\int w_{K}(\lambda) q_{L}(\lambda) d \lambda, \\
F_{L}=\int f(\lambda) q_{L}(\lambda) d \lambda .
\end{gathered}
$$

The vector $\mathbf{F}$ is the contribution due to environmental lighting, including the black level of the projector. The interaction of the spectral responses of the projector and camera are described by $\mathbf{V}$, called the color mixing matrix.

\footnotetext{
${ }^{1}$ Most cameras have a non-linear response function. This may, however, be easily calculated off-line and inverted. The same is true for the non-linear projector response. These responses typically do not change dynamically.

${ }^{2}$ This assumption was also used in [4].
}

Note that this matrix is diagonal for narrow band responses. Typically, the responses of cameras and projectors are wide band and have large overlaps. Thus, we cannot assume $\mathbf{V}$ is diagonal. The matrix $\mathbf{V}$, however, can be calibrated once since it is independent of the surface color. We can account for changes in the surface color by modifying the surface reflectance matrix A. Since $\mathbf{A}$ has only three parameters, we will show we can adapt our model to a changing environment by capturing a single frame.

\section{Dynamic Adaptation}

Equation (3) models projection and capture for a static environment. Over time $t$, both the surface reflectance matrix $\mathbf{A}^{(t)}$ and the environmental lighting $\mathbf{F}^{(t)}$ can change in a dynamic environment. To adapt to this change, our approach is to compensate the image using our current best estimates of the model parameters. As the true parameters change the current estimate becomes invalid. As a result of using this estimate to compensate the projected image, the captured image will differ from the desired image. The difference gives us three values per pixel (RGB) with which to adapt our model parameters.

To adapt in a single frame, it is not possible to fully determine both $\mathbf{A}^{(t)}$ and $\mathbf{F}^{(t)}$. We note that if the projector is capable of performing compensation on textured and colored surfaces, it must be considerably brighter than the environmental lighting. The change in environmental lighting elements $\mathbf{F}^{(t)}$ is typically very small in relation to $\mathbf{V P}$ in equation (3). Thus, we will assume that $\mathbf{F}^{(t)} \approx \mathbf{F}^{(0)}$. We can also assume the projector and camera parameters are fixed so that $\mathbf{V}$ is constant with respect to time.

To model the change in the parameters from an initial $t=0$, we rewrite equation (3) as

$$
\mathbf{C}=\tilde{\mathbf{A}}^{(t)}\left(\tilde{\mathbf{F}}^{(t)}+\tilde{\mathbf{V}} \mathbf{P}\right),
$$

where:

$$
\begin{aligned}
& \tilde{\mathbf{A}}^{(t)}=\mathbf{A}^{(t)}\left(\mathbf{A}^{(0)}\right)^{-1}=\left[\begin{array}{ccc}
\tilde{A}_{R}^{(t)} & 0 & 0 \\
0 & \tilde{A}_{G}^{(t)} & 0 \\
0 & 0 & \tilde{A}_{B}^{(t)}
\end{array}\right], \\
& \tilde{\mathbf{F}}^{(t)}=\mathbf{A}^{(0)} \mathbf{F}^{(t)}=\left[\begin{array}{c}
\tilde{F}_{R}^{(t)} \\
\tilde{F}_{G}^{(t)} \\
\tilde{F}_{B}^{(t)}
\end{array}\right], \\
& \tilde{\mathbf{V}}=\mathbf{A}^{(0)} \mathbf{V}=\left[\begin{array}{ccc}
\tilde{V}_{R R} & \tilde{V}_{R G} & \tilde{V}_{R B} \\
\tilde{V}_{G R} & \tilde{V}_{G G} & \tilde{V}_{G B} \\
\tilde{V}_{B R} & \tilde{V}_{B G} & \tilde{V}_{B B}
\end{array}\right] .
\end{aligned}
$$

We use four uniform calibration images to determine the parameters of equation (4) for $t=0$. The first image we project has a single low value of $\mathbf{P}$ for every pixel. This 
produces a uniform dark projector output image. The three other images are obtained by changing each color input channel $K$ independently so that the output is $\mathbf{P}+\Delta \mathbf{P}$. For example, suppose we only change the red channel. Using the linearity of equation (4) the change in camera irradiance $\Delta \mathbf{C}$ is given by $\Delta C_{K}=\tilde{V}_{L K} \Delta P_{K}$ for $L, K=R, G, B$. Thus, we can determine one column of $\tilde{\mathbf{V}}$ as

$$
\tilde{V}_{R R}=\frac{\Delta C_{R}}{\Delta P_{R}}, \tilde{V}_{G R}=\frac{\Delta C_{G}}{\Delta P_{R}}, \tilde{V}_{B R}=\frac{\Delta C_{B}}{\Delta P_{R}} .
$$

Similarly, the other two columns $\tilde{\mathbf{V}}$ are obtained by changing the input brightness for the green and blue projector channels while keeping the others fixed. This is similar to the method used in [14]. One critical difference is that in [14] the off-diagonal elements were only determined relative to the diagonal elements because the response function was unknown. Here, we have linearized the response of our projector in advance. This allows us to determine all the entries in the matrix $\tilde{\mathbf{V}}$.

The recovery of $\tilde{\mathbf{V}}$ allows us to determine $\tilde{\mathbf{F}}^{(0)}$. Since by construction $\tilde{\mathbf{A}}^{(0)}=\mathbf{A}^{(0)}\left(\mathbf{A}^{(0)}\right)^{-1}=1$, we obtain from equation (4)

$$
\tilde{\mathbf{F}}^{(0)}=\mathbf{C}-\tilde{\mathbf{V}} \mathbf{P} .
$$

Thus, We have computed all terms in equation (4) for $t=0$.

For $t>0$, we do not assume our environment to be static. The environment can be dynamic. For example, the projection system, the surface we project on, or both, may move. It is not practical to repeatedly to project and capture calibration images. Projecting four calibration frames repeatedly is irritating to the user. Instead, our algorithm assumes that we compensate the projected image using our previous estimates $\tilde{\mathbf{A}}^{(t-1)}$ and $\tilde{\mathbf{F}}^{(t-1)}$. If the desired camera irradiance is $\mathbf{C}$, to achieve this irradiance we project

$$
\mathbf{P}=\tilde{\mathbf{V}}^{-1}\left(\left(\tilde{\mathbf{A}}^{(t-1)}\right)^{-1} \mathbf{C}-\tilde{\mathbf{F}}^{(t-1)}\right) .
$$

The actual captured image $\mathbf{C}^{(t)}$ is given by

$$
\mathbf{C}^{(t)}=\tilde{\mathbf{A}}^{(t)}\left(\tilde{\mathbf{F}}^{(t)}+\tilde{\mathbf{V}} \mathbf{P}\right)
$$

where $\tilde{\mathbf{A}}^{(t)}$ and $\tilde{\mathbf{F}}^{(t)}$ are unknown. The compensation results in errors represented by the difference $\mathbf{C}^{(t)}-\mathbf{C}$. Using our assumption that $\tilde{\mathbf{F}}^{(t)} \approx \tilde{\mathbf{F}}^{(0)}$, our new estimate for the channel $K$ of the diagonal reflectance matrix is

$$
\tilde{A}_{K}^{(t)}=\frac{C_{K}^{(t)}}{C_{K}} .
$$

The right hand side of this equation may be computed in real time.

We need only apply the adaptation if we detect a significant change. We can detect changes in the surface reflectance by setting a threshold for the error on the right of equation (9). There is a one-frame lag between detecting the need for adaptation and adapting the model.

Even when the model parameters are accurate, there are limitations to the ability to compensate an image using projection. For example, any projector-based method for creating a display is limited by the dynamic range of the projector and the relative brightness of the environmental lighting. Performance will also vary with the reflectance properties of the material. Purely black, specular, or transparent surfaces create significant challenges for any projector-based method. We will therefore assume that our surface has a significant component of diffuse reflectance, although we do not require it to be matte. Our system has an effective dynamic range equal to the the ratio of the brightest uniform image we can achieve to the darkest. This ratio is large enough to provide the desired appearance of an image projected onto a surface. In fact, current consumer projectors are now bright enough that this is a reasonable assumption in typical home or office settings for a wide variety of common surfaces.

We verify empirically that our adaptive method gives good estimates of $\tilde{\mathbf{A}}$ and $\tilde{\mathbf{F}}$ as compared with the estimates we obtain using full re-calibration. We compared the differences of each component $\tilde{\mathbf{A}} \tilde{\mathbf{F}}$ and $\tilde{\mathbf{A}} \tilde{\mathbf{V}} \mathbf{P}$ in equation (4) using both methods. We sampled 1,400 pixels from captured images where we projected on a wide variety of common surface materials such as paper, cloth and wood. Table 1 shows the average error for the green channel, normalized by 255 (the maximum gray-level). The worst case $\mathbf{P}=255$ was assumed in order to compute the normalized differences between $\tilde{\mathbf{A}} \tilde{\mathbf{V}} \mathbf{P}$ determined using adaptation, and using calibration images.

The average differences of $\tilde{\mathbf{A}} \tilde{\mathbf{F}}$ are less than 0.006 , when comparing adaption to calibration. This is equivalent to about 1.5 gray-levels. The average differences of $\tilde{\mathbf{A}} \tilde{\mathbf{V}} \mathbf{P}$ are less than 0.03 . The differences for the other channels look similar. This confirms that the lighting term dominates the reflectance term, justifying our assumption of effective fixed lighting. Since the total differences are less than a few gray-levels we conclude that our photometric adaptation method gives a good approximation to results achieved with full calibration.

\section{Experimental Results}

We evaluated our algorithm by projecting a variety of images in dynamic environments with colorful surfaces. In our experiments, we used a Sony VPL-CS5 projector with a resolution of $800 \times 600$ pixels and a Sony DXC 950 camera with a resolution of $640 \times 480$ pixels. Images were sent to the projector via an ATI Radeon VE display card and images from the camera were captured using Matrox Meteor 


\begin{tabular}{c|c|c}
\hline materials & $\begin{array}{c}\text { average difference } \\
\text { of } \widetilde{\mathbf{A}} \widetilde{\mathbf{F}}\end{array}$ & $\begin{array}{c}\text { average difference } \\
\text { of } \widetilde{\mathbf{A}} \widetilde{\mathbf{V}} \mathbf{P}\end{array}$ \\
\hline paper & 0.001 & 0.03 \\
cloth & 0.006 & 0.03 \\
wood & 0.003 & 0.01 \\
\hline
\end{tabular}

Table 1: Comparison between the differences of $\tilde{\mathbf{A}} \tilde{\mathbf{F}}$ and $\tilde{\mathbf{A}} \tilde{\mathbf{V}} \mathbf{P}$ with adaptation and with full re-calibration (not adaptation). The average differences (normalized by 255 gray-levels) for the green channel with the worst case $\mathbf{P}=255$ are shown using some materials such as paper, cloth and wood. The differences for the other channels look similar.

\begin{tabular}{c|ccc|ccc}
\hline Desired & \multicolumn{3}{|c|}{ MAX error } & \multicolumn{3}{c}{ RMS error } \\
\cline { 2 - 7 } Brightness & R & G & B & R & G & B \\
\hline 64 & 5 & 4 & 5 & 1.42 & 1.22 & 1.26 \\
128 & 7 & 4 & 9 & 1.71 & 0.88 & 2.24 \\
192 & 10 & 7 & 16 & 2.58 & 1.31 & 3.35 \\
\hline
\end{tabular}

Table 2: Compensation accuracy for various desired brightness values and across channels.

II frame-grabber. Our algorithm ran on a Windows PC with Pentium IV $(1.8 \mathrm{GHz})$ processor.

In our first experiment, the surface is static. The surface is a sheet of paper with various colored patches, as shown in Fig. 3(a). Onto this surface, we projected uncompensated uniform gray images (gray-levels 64, 128 and 192). The images shown Fig. 3(b) were captured from the camera viewing this uncompensated projection. The compensation images that must be projected to achieve a uniform appearance were determined using the model obtained from a full calibration and shown in Fig. 3(c). The result of projecting these images onto the surface is a uniform appearance. The apparent surface pattern has vanished, as shown in Fig. 3(d). Note that making a non-uniform surface appear uniform represents a worst case. This is because it is typically easier to create a patterned appearance since the pattern can mask flaws in the compensation.

The errors are fairly consistent across color channels as we see from Table 2 . The table also shows that the brighter the desired projected image, the larger the errors in compensation. This is largely due to the limited dynamic range of the projector. Our compensation runs in real-time (30 fps).

For our next experiment we created a dynamic environment. Sheets of paper with different arrangements of color patches were placed in a stack. By removing sheets in succession, we created a dynamic surface. The changing surface was captured and the image sequence is shown (from top to bottom) in Fig. 4(a). The top image in Fig. 4(b) shows the captured image when an uncompensated uniform gray image (gray-level 100) is projected on the surface. The remaining images in Fig. 4(b) show the result of projecting (a) surface

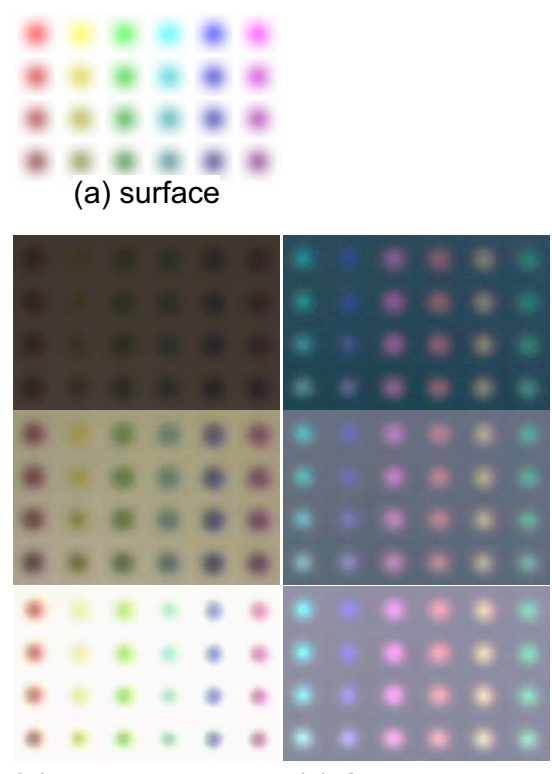

(b) Uncompensated

(c) Compensation

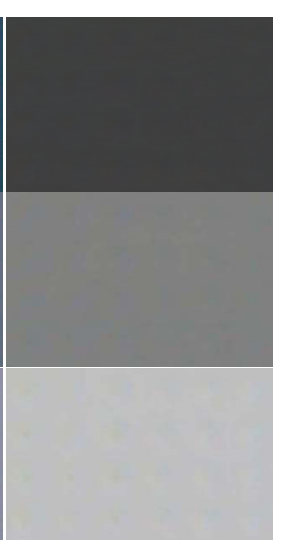

(d) Compensated
Figure 3: Evaluation of our system to make a surface with colored patches appear uniform gray (gray-levels 64, 128, 192) by projection of a compensation image.

the compensation images for the previous surface on current one. The errors in these non-adapted images are significant and thus easily detected in our algorithm. The image sequence in Fig. 4(c) shows the results of compensating using our photometric adaptation algorithm. The first image is compensated using the initial calibration. The following images use the non-adapted images in Fig. 4(b) to determine the surface reflectance matrix A. Even though this adaptation is only an approximation to the model that would be obtained with the calibration images, the resulting compensated images yield comparable results. A key point is that once the surface reflectance matrix is determined, we can project an arbitrary sequence of images in real-time with minimal error. We only need to re-compute the surface reflectance matrix when the surface changes.

Figs. 5 and 6 show the maximum and RMS error respectively, when using photometric adaptation to compute compensation images for the sequence of surfaces in Fig. 4. The compensation is effective and independent of the color of the patch. Comparing the size of the errors from Table 3 with those from Table 2, we find roughly comparable performance. This shows our photometric adaptation achieves good compensation without the need to re-project calibration images. Our method provides real-time compensation for a dynamic surface.

Fig. 7(a) shows a scene that moves during a projection. Using projected calibration images, we see the results obtained in using the recovered model parameters in Fig. 7(b). 


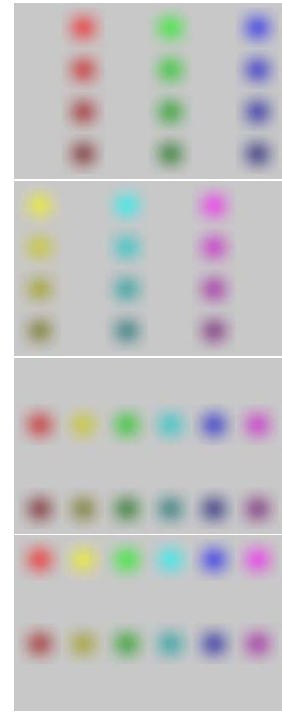

(a) Surface

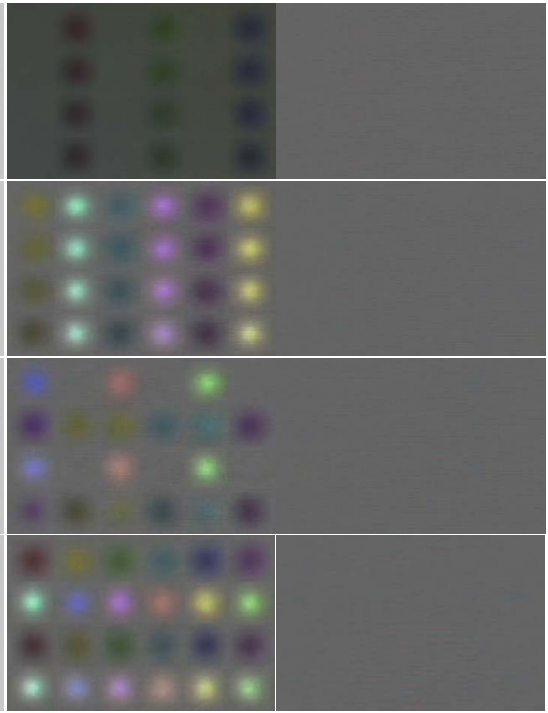

(b) Non-adapted (c) Adapted
Figure 4: Evaluation of our photometric adaptation to create a uniform gray appearance on a sequence of surfaces. The first compensation image is determined using the calibration of the initial surface. Subsequent images detect and use errors in compensation when the surface changes to adapt and provide correct compensation.

If the same model parameters are used to compensate the image as the scene object moves, the result is shown in Fig. 7(c). Not only does the appearance degrade on the moving object, but dis-occlusion of the background creates a ghost where the object was. Our adaptation eliminates the ghosts while correcting the projection on the moving object as shown in Fig. 7(d). This is accomplished in real-time without the need for re-projection of the calibration images.

As projectors become small it becomes possible to embedded them in hand-held devices. This makes it critical to be able to handle the projector system motion as shown in Fig. 8(a). The result of the initial compensation is shown in Fig. 8(b) obtained using calibration images. Without adaptation, the compensation performs poorly as the projector

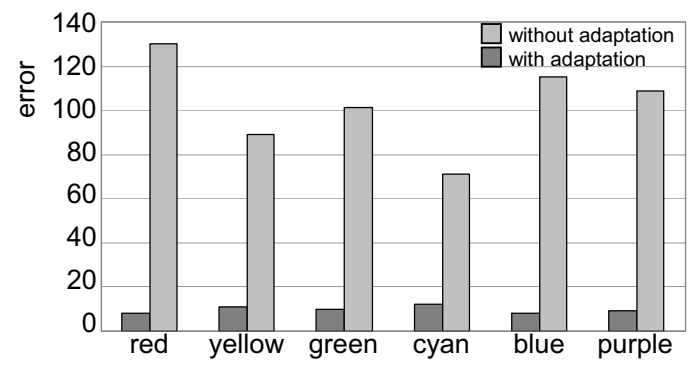

Figure 5: Comparison between maximum errors with and without adaptation for each colored patch.

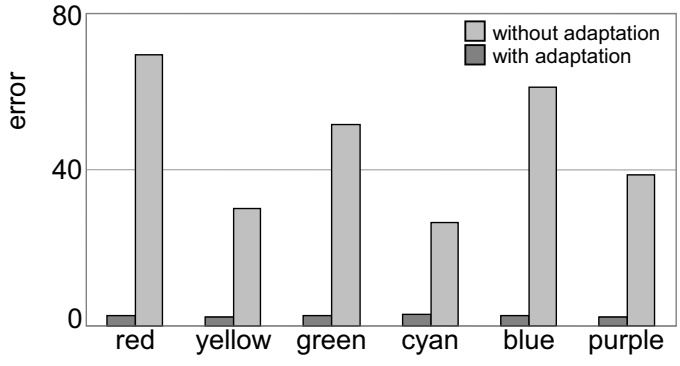

Figure 6: Comparison between RMS errors with and without adaptation for each colored patch.

\begin{tabular}{c|ccc|ccc}
\hline Surface & \multicolumn{3}{|c|}{ MAX error } & \multicolumn{3}{c}{ RMS error } \\
\cline { 2 - 7 } Pattern & R & G & B & R & G & B \\
\hline 1 & 5 & 4 & 5 & 1.03 & 1.05 & 1.11 \\
2 & 7 & 5 & 6 & 1.20 & 1.01 & 1.23 \\
3 & 9 & 5 & 7 & 1.38 & 1.05 & 1.34 \\
4 & 12 & 6 & 10 & 2.04 & 1.01 & 1.59 \\
\hline
\end{tabular}

Table 3: Compensation accuracy across channels using photometric adaptation for a sequence of surfaces.

moves, creating ghosts as shown in Fig. 8(c). Once we apply our adaptation (as in Fig. 8(d)), we obtain a dramatic reduction in artifacts.

Our adaptation method offers even greater advantages for video. As illustrated in Fig. 9(a), part of the scene is shifted up and to the left as the video plays. The captured image shown in Fig. 9(b) makes it clear that using the calibrated model, the original surface pattern can be made to disappear. Fig. 9(c) shows that the surface pattern becomes visible on the building wall after the scene is moved. When our photometric adaptation is applied, the pattern again vanishes. The desired result is shown in Fig. 9(d). We are able to adapt the compensation without re-projection of calibration images. In contrast to model-less methods using dynamic feedback, our compensation is independent of the projected image [14]. Only those parts of the scene which move need to be adapted. More importantly, the adaptation on the moving elements is accomplished in a single frame.

The efficiency of our algorithm enables photometric adaptation at frame-rate. By taking into account reflectance variations on the surface, we ensure that the projected image will be clear. We also are able to rapidly adapt our system when the projected object and the projector are moved.

One application of our results is to aid projector-based augmented reality and tangible interfaces. The use of projection makes it possible to create displays and interfaces on real-world objects without altering the objects or requiring the user to wear special equipment. Fig. 10 shows direct annotation of objects on a desk. This is inspired by the concept of an augmented desk interface [15], which provide us to detect and recognize the user interaction with tangible 


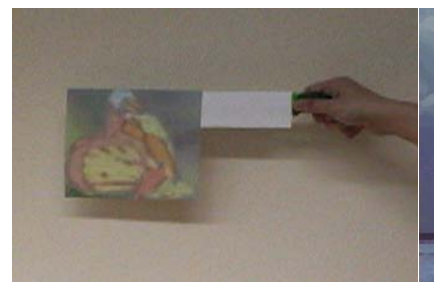

(a) Surface

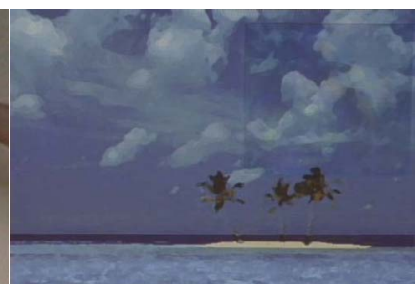

(b) Compensated

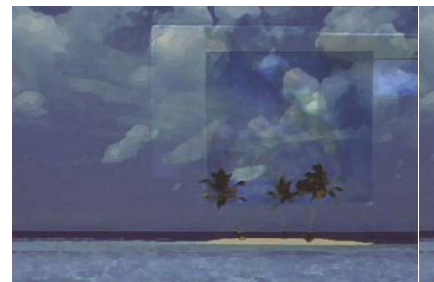

(c) Non-adapted

(d) Adapted

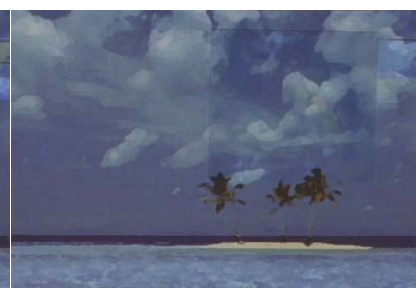

Figure 7: Results showing projection onto a scene in which an object moves. (a) A picture moved in front of background. (b) Initial compensation using calibration. (c) Motion without compensation. (d) The result of adaptive compensation using our algorithm.

objects. We show, for example, relevant information can be projected onto the objects. When users place an envelope on the desk, the annotations regarding an envelope appear on it. As shown in Fig. 11, the projected annotations are essentially invisible if the projection is not adapted to the object's reflectance. Once adaptation is applied, the annotations become clear.

\section{Conclusion}

We have presented a method for photometric adaptive projection in dynamic environments. Our projector-camera design consists of a camera and beam splitter, which attaches to an off-the-shelf projector. The co-axial design makes geometric calibration scene-independent. Our photometric adaptation technique combines an efficient and accurate photometric model with dynamic feedback. In this paper we have described adapting the three parameters associated with changing surface reflectance. If the surface reflectance remains constant but the lighting changes significantly, it is possible to modify our method to adapt to this kind of change. In future work, we hope to investigate adapting to changes in reflectance and lighting together, using the errors from a sequence of frames. Our current method moves beyond the limits of a static environment to make real-time color compensation in a dynamic environment possible.

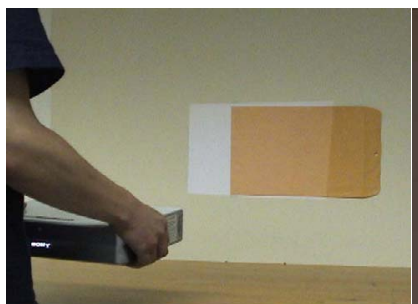

(a) Surface

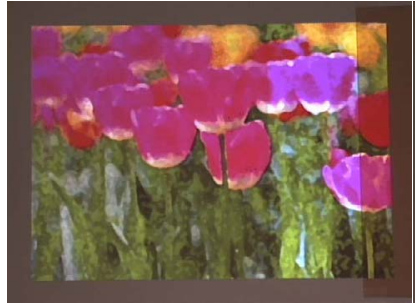

(c) Non-adapted

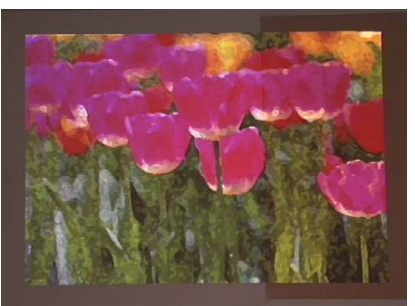

(b) Compensated

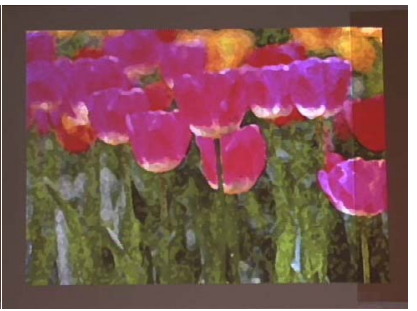

(d) Adapted
Figure 8: Compensation results where the projector is moved. (a) Moving projector. (b) Initial compensation results. (d) results without adaptation. (c) Result using our photometric adaptation.

\section{Acknowledgments}

This work was conducted at the Columbia Vision and Graphics Center in the Department of Computer Science at Columbia University. It was supported by an NSF ITR Award (No.IIS-00-85864). The authors are grateful to Ko Nishino, Sujit Kuthirummal and other lab members for valuable discussions on early drafts.

\section{References}

[1] D. Bandyopadhyay, R. Raskar, and H. Fuchs. Dynamic shader lamps: Painting on movable objects. In Proc. of International Symposium on Augmented Reality, pages 207-216, 2001.

[2] I.E. Bell. Neutralizing paintings with a projector. In Proc. of SPIE/IS\&T, volume 5008, pages 560-568, 2003.

[3] O. Bimber, A. Emmerling, and T. Klemmer. Embedded entertainment with smart projectors. IEEE Computer, 38(1):48-55, 2005.

[4] D. Caspi, N. Kiryati, and J. Shamir. Range imaging with adaptive color structured light. IEEE Trans. on Pattern Analysis and Machine Intelligence, 20(5):470-480, 1998.

[5] C. Cruz-Neira, D.J. Sandin, and T.A. DeFanti. Surround-screen projection-based virtual reality: The design and implementation of the cave. In Proc. of SIGGRAPH, pages 135-142, 1993.

[6] C. Funayama, M. Tsukada, and J. Tajima. Development of projector color reproduction adapted to wall color. In In Proc. of Color Forum Japan, pages 103-106, 2002.

[7] M.D. Grossberg, H. Peri, S.K. Nayar, and P.N. Belhumeur. Making one object look like another: Controlling appearance using a projector-camera system. In Proc. of IEEE Conference on Computer Vision and Pattern Recognition, pages 452-459, 2004.

[8] M. Herald, I.R. Judson, and R.L. Stevens. Introduction to building projection-based tiled display systems. IEEE Computer Graphics and Applications, 20:22-28, 2000. 


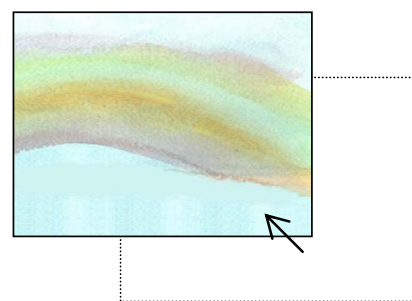

(a) Surface

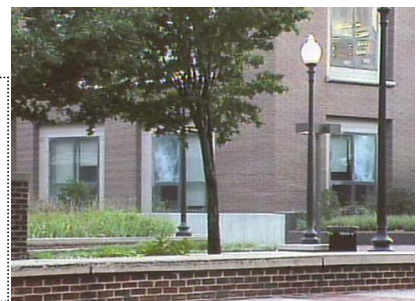

(b) Compensated

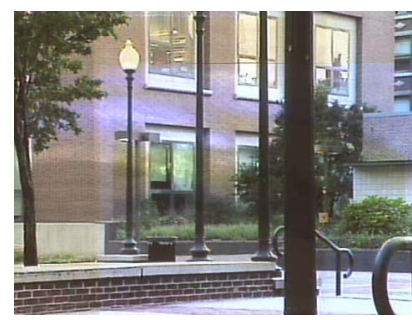

(c) Non-adapted

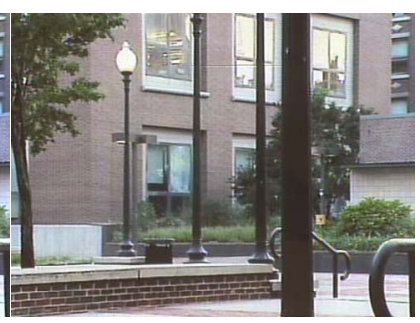

(d) Adapted
Figure 9: Projecting video on a moving painting (a) Illustration showing the painting. (b) Results using projection of calibration images. (c) Results without adaptation. (d) Video compensated for scene motion using photometric adaptation.

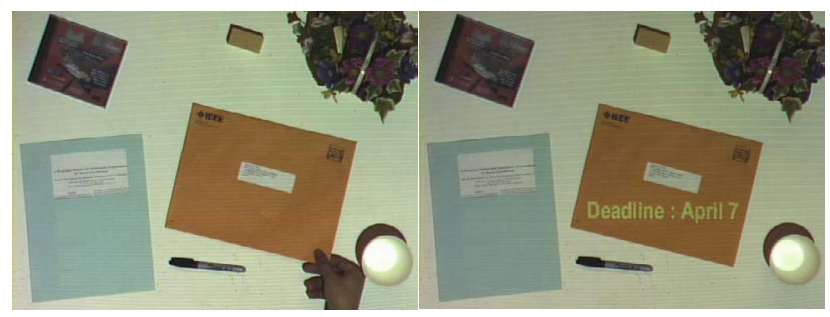

Figure 10: Direct annotation with adaptation. Relevant information regarding an object, such as a piece of mail, is projected directly onto the object.

[9] C. Jaynes, S. Webb, R. Steele, M. Brown, and B. Seales. Dynamic shadow removal from front projection displays. In Proc. of IEEE Visualization, pages 152-157, 2001.

[10] R. Kjeldsen, C. Pinhanez, G. Pingali, J. Hartman, T. Levas, and M. Podlaseck. Interacting with steerable projected displays. In Proc. of International Conference on Automatic Face and Gesture Recognition, pages 12-17, 2002.

[11] K. Li and Y. Chen. Optical blending for multi-projector display wall system. In Proc. of IEEE Lasers and Electro-Optics Society, pages 281-282, 1999.

[12] A. Majumder, Z. He, H. Towles, and G. Welch. Color calibration of projectors for large tiled displays. In Proc. of IEEE Visualization, pages 102-108, 2000.

[13] N. Nakamura. Active projector: Image correction for moving image over uneven screens. In Proc. of User Interface Software Technology, pages $1-2,2002$.

[14] S.K. Nayar, H. Peri, M.D. Grossberg, and P.N. Belhumeur. A projection system with radiometric compensation for screen imperfections. In Proc. of ICCV Workshop on Projector-Camera Systems, 2003.

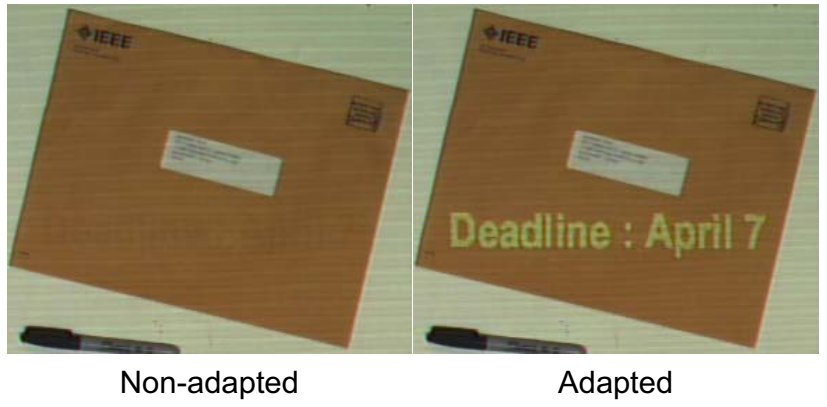

Figure 11: Comparison with and without adaptation. The annotation is essentially invisible due to the color of the envelope. After applying photometric adaptation, the projected annotation becomes clearly visible.

[15] K. Oka, Y. Sato, and H. Koike. Real-time tracking of multiple fingertips and gesture recognition for augmented desk interface systems. IEEE Computer Graphics and Applications, 22(6):64-71, 2002.

[16] C. Pinhanez. The everywhere displays projector: A device to create ubiquitous graphical interfaces. In Proc. of Ubiquitous Computing, pages 12-17, 2001.

[17] B. Piper, C. Ratti, and H. Ishii. Illuminating clay: A 3-d tangible interface for landscape analysis. In Proc. of CHI, pages 355-362, 2002.

[18] R. Raskar, J. Baar, P. Beardsley, T. Willwacher, S. Rao, and C. Forlines. ilamps: Geometrically aware and self-configuring projectors. ACM Trans. on Graphics, 22(3):809-818, 2003.

[19] R. Raskar and P. Beardsley. A self correcting projector. In Proc. of IEEE Computer Vision and Pattern Recognition, pages 504-508, 2001.

[20] R. Raskar, P. Beardsley, J. Baar, J. Wang, P. Dietz, D. Leigh, and T. Willwacher. Interacting with a self-describing world via photosensing wireless tags and projectors. In Proc. of ACM SIGGRAPH, pages 406-415, 2004.

[21] R. Raskar, G. Welch, M. Cutts, A. Lake, L. Stesin, and H. Fuchs. The office of the future: A unified approach to image-based modeling and spatially immersive displays. In Proc. of SIGGRAPH, pages 179188, 1998.

[22] R. Sukthankar, T.J. Cham, and G. Sukthankar. Dynamic shadow elimination for multi-projector displays. In Proc. of IEEE Computer Vision and Pattern Recognition, pages 151-157, 2001.

[23] S. Tominaga. Surface identification using the dichromatic reflection model. IEEE Trans. on Pattern Analysis and Machine Intelligence, 13(7):658-670, 1991.

[24] J. Underkoffler, B. Ullmer, and H. Ishii. Emancipated pixels: Realworld graphics in the luminous room. In Proc. of SIGGRAPH, pages 385-392, 1999.

[25] R. Yang, D. Gotz, J. Hensley, H. Towles, and Brown M. Pixelex: A reconfigurable multi-projector display system. In Proc. of IEEE Visualization, pages 167-174, 2001.

[26] T. Yoshida, C. Horii, and K. Sato. A virtual color reconstruction system for real heritage with light projection. In Proc. of International Conference on Virtual Systems and MultiMedia, pages 825831, 2003. 